\title{
e-Tutor, acompañamiento estudiantil digital en la Universidad Tecnológica del Sureste de Veracruz
}

\section{e-Tutor, digital student support at the Technological University of Southeast Veracruz}

\author{
KATT-MORALES, Luz Alondra, MORALES-REYES, Eunice y GOMEZ-MANUEL, Esbeydi \\ Universidad Tecnológica del Sureste de Veracruz
}

ID $1^{\text {er }}$ Autor: Luz Alondra, Katt-Morales / ORC ID: 0000-0002-9982-8718, Researcher ID Thomson: S-6606-2018, CVU CONACYT ID: 412698

ID $1^{\text {er }}$ Coautor: Eunice, Morales-Reyes / ORC ID: 0000-003-0658-6957, Researcher ID Thomson: S-4739-2018

ID $2^{\text {do }}$ Coautor: Esbeydi, Gomez-Manuel / ORC ID: 0000-003-0765-3402, Researcher ID Thomson: G-2859-2019, CVU CONACYT ID: 599053

DOI: $10.35429 / J E S C .2019 .9 .3 .19 .24$

Recibido: 10 de Junio, 2019; Aceptado 30 de Septiembre, 2019

\section{Resumen}

La creación del Programa Institucional de Tutorías y Plan de Acción Tutorial, surge como estrategia de la Universidad Tecnológica del Sureste de Veracruz para contribuir en el acompañamiento estudiantil de los jóvenes estudiantes de nivel superior. El propósito de la incorporación de las tecnologías de información está orientado a enfrentar oportunamente los fenómenos de deserción, rezago e inclusive reprobación observados. Partiendo de la revisión de literatura, análisis de indicadores institucionales, así como el uso de la metodología de sistematización de experiencias, se da cabida al acompañamiento estudiantil electrónico, lo que permite evaluar resultados por medio de las estadísticas presentadas, siendo favorables, con ello la toma de decisiones es oportuna, identificando casos de reprobación ofreciendo asesorías académicas, mitigando hasta en un $20 \%$ la deserción escolar asimismo se resalta la innovación lograda en la gestión de la información, permitiendo su obtención en tiempo real.

Acompañamiento, Tutorías, Digital

\begin{abstract}
The creation of the Institutional Tutoring Program and Tutorial Action Plan, arises as a strategy of the Technological University of the Southeast of Veracruz to contribute to the student support of young students of higher level. The purpose of the incorporation of information technologies is oriented to timely address the phenomena of desertion, lag and even disapproval observed. Starting from the literature review, analysis of institutional indicators, as well as the use of the methodology of systematization of experiences, it is possible to accommodate electronic student accompaniment, which allows evaluating results through the statistics presented, being favorable, thereby Decision-making is timely, identifying cases of failure to offer academic advice, mitigating up to $20 \%$ of school dropouts also highlights the innovation achieved in the management of information allowing its obtaining in real time.
\end{abstract}

Accompaniment, Tutoring, Digital

Citación: KATT-MORALES, Luz Alondra, MORALES-REYES, Eunice y GOMEZ-MANUEL, Esbeydi. e-Tutor, acompañamiento estudiantil digital en la Universidad Tecnológica del Sureste de Veracruz. Revista de Ciencias de la Educación. 2019. 3-9: 19-24

\footnotetext{
* Correspondencia del Autor (luz.katt@utsv.edu.mx)

$\dagger$ Investigador contribuyendo como primer autor.
} 


\section{Introducción}

El acompañamiento estudiantil es una estrategia que se ha ido adoptado en el medio universitario como respuesta a los elevados índices de deserción, reprobación en instituciones de educación superior, así mismo constituye una alternativa para la revisión de estilos de aprendizaje, técnicas de estudio y como guía en el medio universitario y académico.

$\mathrm{Al}$ aplicar el acompañamiento de forma institucional, se estable de forma coherente y sistemática, de tal forma que contribuya efectivamente en el alcance de los objetivos de la educación superior como son: disminuir índice de deserción, reprobación e incrementar la eficiencia terminal.

La tutoría es un proceso de acompañamiento, cuyo objetivo radica en guiar al tutorado en el medio universitario $\mathrm{y}$ académico, para facilitar la autonomía en su formación integral, sin embargo para lograrlo, el tutor debe tener formación que le permita conocer el comportamiento del alumno, la metodología de trabajo tutorial para identificar y atender los problemas que afronte el estudiante en el medio universitario que lo rodea, como menciona Sagastume, el tutor puede implementar estrategias y técnicas, con la finalidad de tener mayor participación e interacción por parte delos estudiantes del curso; por lo que tener presencia diaria, promover la comunicación, habilidad para atender dudas, mantener informado a los estudiantes, utilizar diferentes medio de comunicación así como establecer un ambientes confortable y de confianza ocasiona la interacción. (Sagastume, et.al, 2018)

Con el creciente cambio tecnológico, y el uso en las instituciones de educación superior, se hace preciso generar un medio electrónico que fortalezca el proceso de tutoría, apoyando a los docentes en las canalizaciones, seguimiento académico o como medio de comunicación con los estudiantes. Este trabajo se centra en evidenciar las fortalezas del acompañamiento estudiantil apoyado de las TI, dado que permite articular acciones de atención y acompañamiento de forma oportuna, tras identificar estados vulnerables y situaciones de riesgo académico en la Universidad Tecnológica del Sureste de Veracruz (UTSV).
Al conjugar el acompañamiento estudiantil y las tecnologías de la información surge etutor, buscando dar seguimiento puntual a las acciones tutoriales implementadas, promover comunicación y el acercamiento entre tutorados y tutor, monitorear la situación académica de cada estudiante para evitar reprobación y ofrecer apoyos a través de asesorías académicas, con la entrevista inicial identificar riesgos y estados de vulnerabilidad que detonen deserción escolar. La deserción se entiende como el abandono escolar por parte de los estudiantes por causa de una combinación de factores como el perfil vocacional que define los Intereses, aptitudes, personalidad y capacidades que tiene una persona con respecto a la elección de una carrera universitaria, otros factores como los económicos, familiares, sociales y/o personales hacen parte de este contexto.(Calvache, et al,. 2018)

\section{Descripción del Método}

El presente trabajo se llevó a cabo en el Universidad Tecnológica del Sureste de Veracruz, con el objetivo de comprender el impacto del tutor. La función del tutor es sumamente importante, aún en nivel superior, ya que el joven se encuentra en un proceso de crecimiento intrapersonal, de madurez, autonomía y de toma de decisiones, por ende el tutor adquiere la labor de brindar apoyo, como guía en el medio universitario o académico, de ahí la necesidad de implementar nuevas experiencias de aprendizaje, es por ello que se genera un plan institucional de tutorías (PIT), ya que se pretende fomentar la formación integral del estudiante, acompañamiento en el medio universitario que permita el desarrollo de habilidades, aptitudes y actitudes por medio del apoyo académico y fortalecimiento de valores, así mismo, con el Programa de Acción Tutorial (PAT), se busca potenciar el desarrollo de habilidades, aptitudes y actitudes por medio del apoyo académico y fortalecimiento de valores a través de actividades de integración, cursos, talleres o asesorías requeridas, lo anterior en apego al manual nacional de tutorías de la coordinación de universidades tecnológicas y politécnicas.

La realización del trabajo se llevó a cabo bajo la siguiente metodología: 


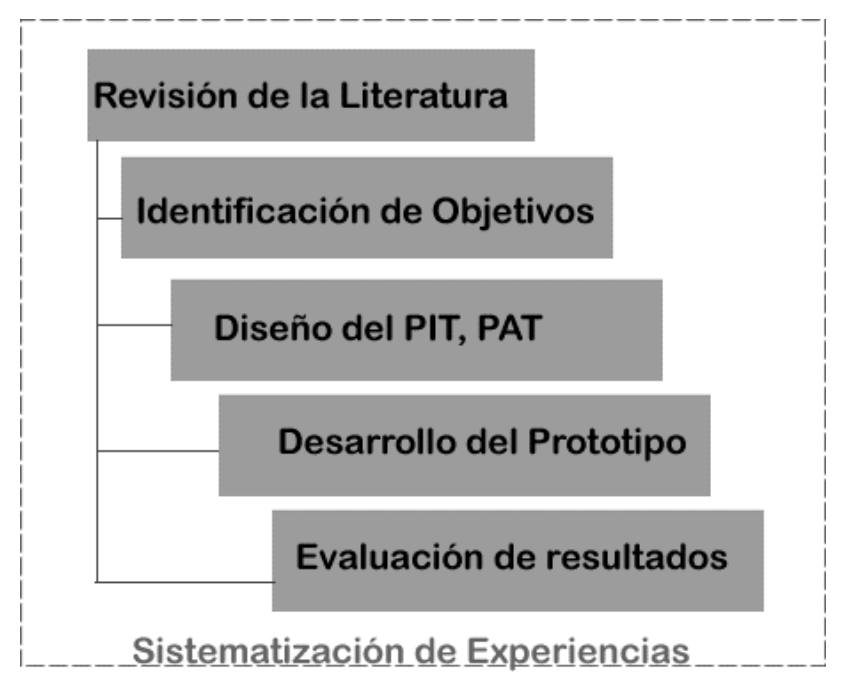

Figura 1 Metodología de trabajo e-tutor

\section{Revisión de la literatura}

En las Universidades Tecnológicas, es importante que se brinde al joven universitario los saberes ; Saber saber, saber hacer, el saber ser, siendo los dos primeros con enfoque cognoscitivo y el tercero con el enfoque humanista, desde dos vertientes, la preparación externa del alumno hacia el campo laborar, como la interna, del joven con el medio universitario.

Es por ello que en la UTSV se busca establecer acompañamiento universitario, que les permita al estudiante y al tutor, reconocer los obstáculos, dificultades familiares, personales, sociales o económicas que puedan afectar su desempeño académico, así mismo identificar índices de reprobación de tal forma que se puedan tomar acciones, como lo menciona Alarcón (Alarcón, et al., 2018) dar solución por medio de la correspondencia entre el docente y acciones definidas que promuevan la permanencia del estudiante en sus objetivos profesionales.

Retomando las palabras de Saldarriaga la tutoría universitaria, se podría traducir en un modelo de tutoría personalizada, ya que a los alumnos se les asigna tutor, quien se encargará de la evolución académica, lo brindará un respaldo desde la perspectiva de los alumnos, debido a que se convierte en un elemento que promueve y afianza la seguridad de tener un guía que estará predispuesto a solventar y dilucidar dudas, o dificultades, tanto en el plano académico como personal. (Saldarriaga, et. al, 2018)
El "propósito del acompañamiento estudiantil está orientado a enfrentar los graves fenómenos de deserción, fracaso académico y rezago", en con secuencias promover el éxito académico en los estudiantes. (Barbosa, 2018) En el plano académico resulta de gran beneficio el respaldo del docente- tutor, para promover un aprendizaje significativo con orientación respecto a técnicas de estudio, actividades de lectura comprensiva, estrategias de metacognición. Es importante mencionar que el acompañamiento estudiantil en la versión digital requiere de metahabilidades, dado la evolución en la educación virtual; sin embargo como menciona Jasso Peña, se muestra actitud positiva por parte de los jóvenes hacia el papel del desarrollo de las metahabilidades, lo que demuestra idoneidad del acompañamiento virtual. (Jasso peña, et. al 2019)

\section{Identificación de Objetivos}

Atender mayor número de jóvenes, por medio del acompañamiento estudiantil empleando tecnologías de la información, abriendo un canal de comunicación con la comunidad universitaria, permitiendo realizar acciones conjuntas de atención de forma oportuna, identificando estados vulnerables y situaciones de riesgo académico en la UTSV para disminuir índices de deserción y reprobación.

\section{Diseño de Plan Institucional de Tutorías (PIT) y Plan de Acción Tutorial (PAT).}

Para lograr el acompañamiento estudiantil se establecen: el programa institucional de tutorías y el plan de acción de tutorial, donde se muestran los ejes rectores, actores involucrados y los roles establecidos para lograr la consolidación.

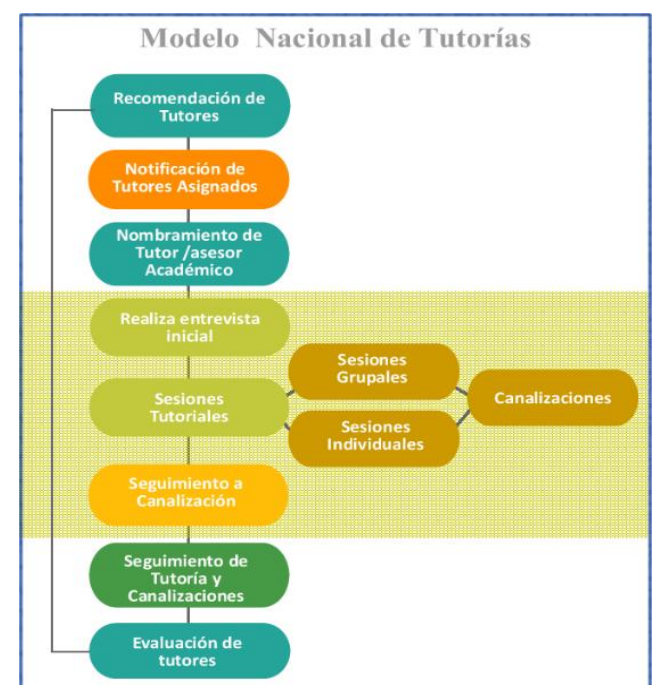

Figura 2 Plan Institucional de Tutoría

KATT-MORALES, Luz Alondra, MORALES-REYES, Eunice y GOMEZ-MANUEL, Esbeydi. e-Tutor, acompañamiento estudiantil digital en la Universidad Tecnológica del Sureste de Veracruz. Revista de Ciencias de la Educación. 2019 
Para la ejecución del PIT, intervienen diversos actores:

EL coordinador de Tutorías: Es quien detona el proceso, dado que recomienda a los docentes que podrían fungir como tutores en el cuatrimestre inmediato posterior, derivado de los resultado de su evaluación, una vez asignados los tutores.

Responsable de Tutorías: Es el encargado del trabajo conjunto entre alumnos, tutores y servicios estudiantiles.

Tutor: Es uno de los actores principales, ya que tiene contacto directo con la comunidad estudiantil, sirve como guía académico y en el medio universitario, por lo que debe tener o adquirir habilidades para trabajo en equipo, detección de necesidades.

Servicios Estudiantiles: Son los encargados de ofrecer diversos servicios a los jóvenes estudiantes, como son: académico, médico, psicopedagógico, cultural y deportivo con la finalidad de brindar atención integral.

Alumnos: Son los actores principales dado que el programa es dirigido a su atención, acompañamiento y lograr la eficiencia terminal, ellos pueden solicitar apoyo individual a su tutor o a algún servicio por medio de la plataforma.

Para llevar a cabo el proceso de tutorías se emplea el procedimiento tutorial, en el cual se establecen las actividades y la interacción de cada actor desde la perspectiva de acompañamiento estudiantil.

\section{Desarrollo del prototipo}

La tutoría digital por medio de una plataforma web permite el acceso a la información en cualquier lugar en cualquier momento, dado que puede accederse desde cualquier navegador web y la información está contenida en una base de datos, teniendo diversos usuarios, de acuerdo a los roles establecidos en el PIT

EL coordinador de Tutorías: Agrega en el sistema y asigna al grupo tutorado, emite nombramientos y gestiona cursos para actualización y formación de tutores.
Responsable de Tutorías: En plataforma tiene acceso a las entrevistas iniciales, estadísticas de género, asistencias a tutorías, canalizaciones y seguimiento a servicios estudiantiles.

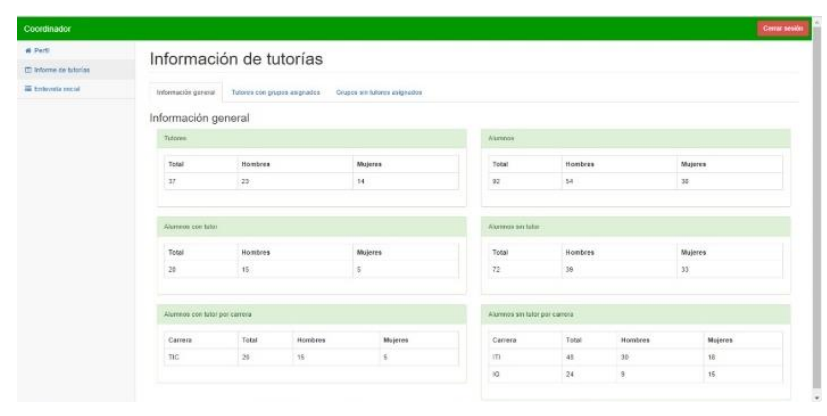

Figura 3 Informe de tutorías

Tutor: Es uno de los actores principales, ya que tiene contacto directo con la comunidad estudiantil, sirve como guía académico y en el medio universitario, por lo que debe tener o adquirir habilidades para trabajo en equipo, detección de necesidades. Mediante el uso de la plataforma, el docente podrá realizar la entrevista inicial que le permitirá conocer las situaciones de riesgo y darle atención oportuna al solicitar las canalizaciones a los servicios, ya sean: médico, académico, becas o psicopedagógica para evitar la deserción o reprobación estudiantil.

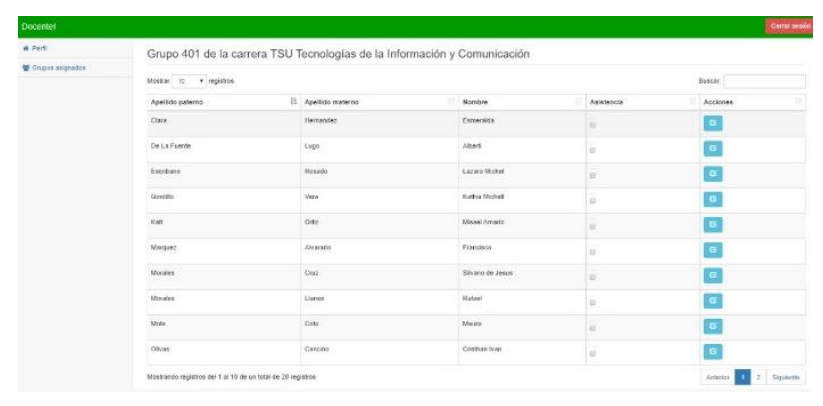

Figura 4 Asistencia a sesiones

Servicios Estudiantiles: Dan seguimiento a las canalizaciones o solicitudes vía plataforma, apoyando a los tutores.

Directores: Son los encargados monitorear las asistencias de alumnos y solicitudes de canalizaciones por parte de los tutores, en su momento canalizar a los estudiantes a algún servicio por medio del responsable de tutorías.

Alumnos: Solicitar apoyo individual a su tutor o a algún servicio por medio de la plataforma. 


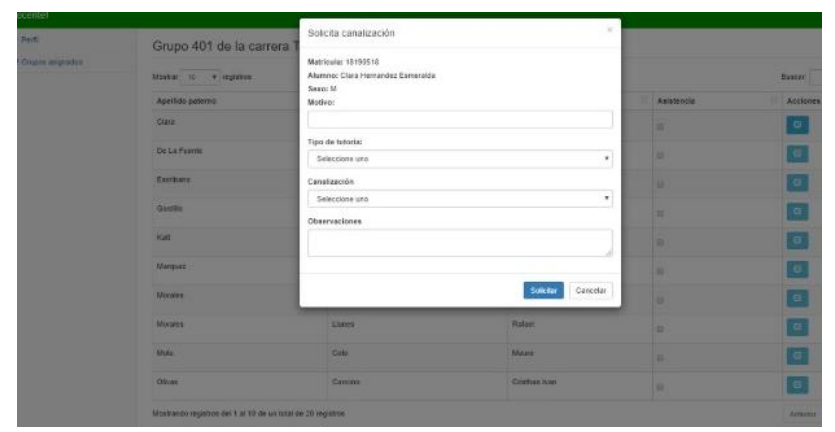

Figura 5 Solicitud de canalizaciones

\section{Resultados}

La inclusión digital en el Programa Institucional de Tutoría permite mejorar el proceso administrativo, desde tres puntos de vista:

Organización: Uso de información a cualquier hora en cualquier momento, contribuyendo en el seguimiento a los estudiantes aún en la ausencia del tutor, dando respuestas oportunas ante emergencias, así mismo identificar alumnos vulnerables lo que ha permitido proporcionar becas y aumentar en un $300 \%$ en comparación con el año anterior.

Control: Los tutores pueden realizar la entrevista inicial, siendo la encuesta de identificación del estudiante, proporcionando una ficha académica, económica y social, además al tomar asistencia vía plataforma, les proporciona historial de sesiones, con lo que puede identificar ausencias continúas y tomar medidas para evitar reprobación.

Toma de decisiones: Al tener informes de indicadores, por género y número, coadyuva a dar respuestas inmediatas, con la finalidad de apoyar en la disminución de índices de reprobación al solicitar y monitorear canalizaciones, al identificar situaciones de vulnerabilidad proporcionar apoyo con distintos servicios estudiantiles. Cabe mencionar que los indicadores y mejorías son identificados a partir de la prueba beta, en este cuatrimestre se espera la implantación para realizar la medición efectiva.

\section{Agradecimiento}

Mi agradecimiento para las personas que intervinieron en el desarrollo del proyecto, tutores, coordinador de tutorías, responsable de tutorías, alumnos y sobre todo a mis alumnos colaboradores, ya que sin el apoyo de algunos de ellos esto no sería posible.

\section{Conclusiones}

Luego de implementar la sistematización de experiencias, se hace un recuento de la intervención del acompañamiento estudiantil, desde el ámbito social, económico y académico, es importante que en las Universidades se brinde una formación humanista a la par del desarrollo académico, lo que le permitirá al estudiante identificar obstáculos o dificultades, en el ámbito familiar, personal, social o económico que afecten su desempeño académico, debido a ello, al realizarse el proceso de tutorías permite brindar una posible solución por medio de la interacción entre tutor y alumno, haciendo uso de los servicios estudiantiles con los que cuenta, ofreciendo acciones que promuevan la permanencia del estudiante.

Al implementar el acompañamiento digital, se resaltan la innovación en la gestión dela información, permite establecer sinergia entre diversas área de apoyo que brindan servicio al estudiante, en vez de trabajar dispersos, la plataforma permite trabajo colaborativo detonando seguimiento oportuno en tiempo real ante situaciones que puedan influir en la deserción o reprobación.

\section{Referencias}

Alarcón Barcia Laura Andrea, carrion Esmeralda, Zambrano Tania. (2018) El acompañamiento estudiantil en el desempeño académico de los alumnos. Revista Científica del ITSUP. Vol. 2 Núm. 13 (2018): Alcances de las Tecnologías de la Información y las Comunicaciones: Educación, Salud y Sociedad

Barbosa Gómez, F., Castillo Florez, N., \& Vásquez Truisi, M. (2018). Estrategia de Acompañamiento y Seguimiento Estudiantil en la Universidad del Valle (Colombia). Congresos CLABES. Recuperado a partir de https://revistas.utp.ac.pa/index.php/clabes/articl e/view/2040 
Calvache Fernández Leidy Carolina, Alvarez Vallejo Valentina, Triviño Arbeláez Jorge Ivan, Quiceno Restrepo, Claudia, Pulgarin Giraldo Robinson(2018). Técnicas de Minerías de Datosa para la identificación de patrones de deserción estudiantil como apoyo a las estrategias de SARA (Sistema de Acompañamiento para el Rendimiento Académico). Revista de la Universidad Tecnológica de Panamá. Congresos CLABES. Recuperado a partir de mayo https://revistas.utp.ac.pa/index.php/clabes/articl e/view/2021

Cárdenas Rodríguez Magaly, Méndez Hinojosa Luz Marina, González Ramírez Mónica Teresa. (2014) Evaluación del desempeño docente, estrés y burnout en profesores universitarios. Revista actualidades investigativas en educación. Volumen 14, Número 1 Enero - Abril pp. $1-22$. https://www.scielo.sa.cr/pdf/aie/v14n1/a05v14n 1.pdf

García Heredia Francisco Javier, Ramírez Martínez Rosana, Portillo Sánchez Rebeca, Saenz Villela Jesús Manuel. (Agosto, 2016)Implicación de la educación en la sociedad del conocimiento, información y comunicación. CULCyT, Año 13, No 59, Especial No. 1, P 358365.

Garzón Daza Cecilia (2018) La gestión educativa en la educación superior de cara a la consejería estudiantil. Boletín Redipe, ISSNe 2256-1536, Vol. 7, №. 8, 2018 (Ejemplar dedicado a: Ethics, management evaluation educational), págs. 98-112

Jaramillo Correa Carolina, Ordóñez Torres Costanza, González Correal Alejandra María, León Neira María Patricia, Curiel Huérfano Mariela Josefina, Palacios Molina Ingrid Julieth y Barrera Ferro Oscar David (2018) Formulación del programa de prevención de la deserción: Articulación de acciones en un programa transversal de acompañamiento para la Pontificia Universidad Javeriana. Congresos CLABES. Recuperado a partir de mayo https://revistas.utp.ac.pa/index.php/clabes/articl e/view/1978
Jasso Peña Felipe de Jesús, Tamez Solís José Porfirio,Torres Reyes José Antonio. (2019) Metahabilidades en información y evolución conceptual en la educación virtual. IE Revista de Investigación Educativa de la REDIECH, 10(18), pp. 23-42. http://dx.doi.org/10.33010/ie_rie_rediech.v10i1 8.165

Sagastume Flor, Morales Miguel, Amado Héctor, Hernández Rocael. (2018)La importancia del tutor en los cursos virtuales: experiencia, buenas prácticas y recomendaciones. Proceedings of the Digital World Learning Conference CIEV 2018

Saldarriaga Villamil Kasandra Vanessa, Amor Almeida María Isabel.(2018) las tutorías y la formación profesional: caso de estudio Universidad Técnica de Manabí, Portoviejo,Ecuador.recuperado en: http://ecociencia.ecotec.edu.ec/articulo?ida=17 0

Universidad Santo Tomas. (2013). Programa de desarrollo estudiantil. Bogotá, D.C.: Universidad Santo Tomas 